\title{
Programa de normalización de estudios previos y control de calidad en las intervenciones: morteros empleados en construcciones históricas
}

Formulación y características $2^{a}$ parte

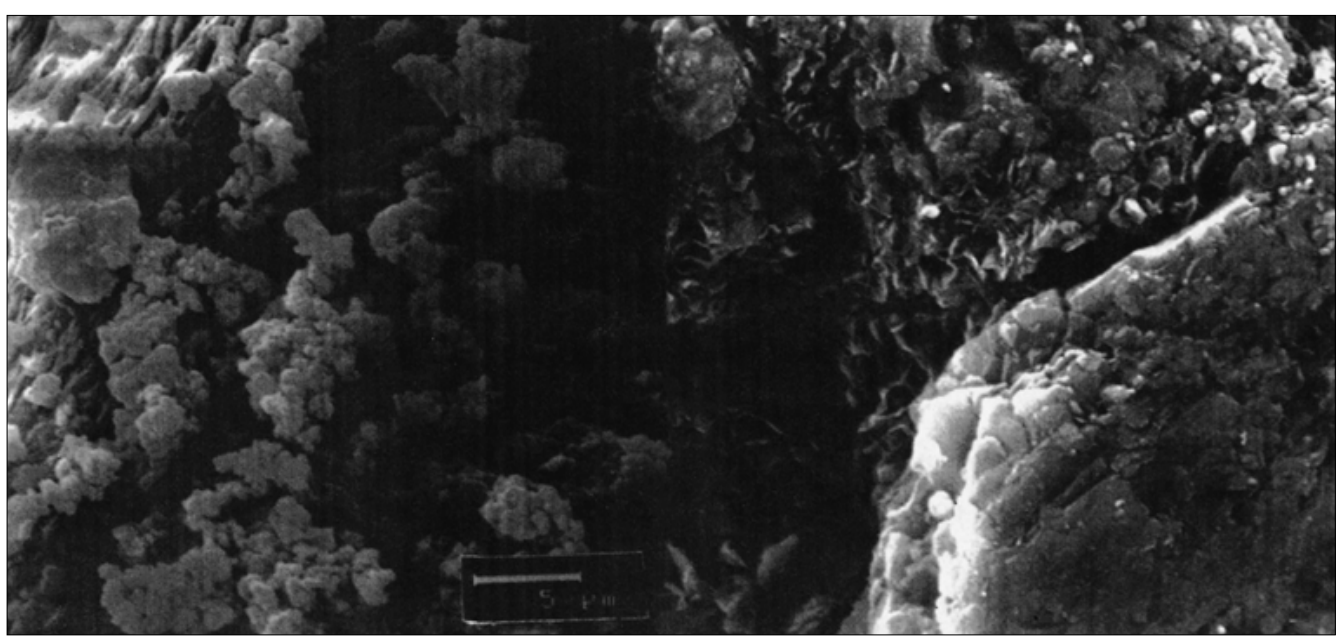

Desarrollo de geles alrededor de los áridos. Reacción árido-álcalis

\section{Esther Ontiveros Ortega}

Departamento de Análisis del IAPH

Resumen

Se continúa con el Programa de Normalización de Estudios Previos y Control de Calidad de las Intervenciones, centrado en la Normalización de Técnicas de Análisis aplicadas a morteros de construcciones históricas. En este artículo se exponen los distintos procesos físico-químicos que se generan en el seno de estos materiales, incluyéndose: hidratación, endurecimiento y reacciones árido-álcalis.

\section{Palabras clave}

Mortero / Fraguado / Hidratación / Endurecimiento / Carbonatación / Sales solubles / Deterioro / Reacciones árido-alcalis.

\section{INTRODUCCIÓN}

En el estudio de morteros antiguos, así como en los de reparación, se deben tener en cuenta las siguientes consideraciones :

- Investigación paralela del mortero antiguo y del de restauración,

- Elección adecuada de la mezcla a utilizar en el mortero de intervención,

- Identificación de los parámetros fisicoquímicos y

- Control de calidad del nuevo mortero.

El conocimiento de la existencia y proporción de los distintos compuestos que aparecen en estos materiales, así como la forma de prepararlos, ingredientes utilizados, producto final obtenido, condiciones de exposición, etc, tienen gran importancia en el campo de la conservación de edificios y obras de carácter histórico. 
Con respecto a las características que debe presentar un mortero de reparación hay que tener en cuenta la compatibilidad del material con el que va a estar en contacto; esto obliga a estudiar la reactividad potencial de los materiales originales (piedra, ladrillo o mortero antiguo) con los componentes de la fase intersticial del mortero de intervención. De igual manera debe presentar fácil trabajabilidad, rapidez de colocación en ambientes secos y húmedos, características mecánicas y térmicas semejantes a los de la fábrica original y mínimo contenido en sales solubles.

Los morteros que se utilizan actualmente para las intervenciones (con cemento portland en su composición) producen sales sódicas y alcalinas, e incluso pueden producir eflorescencias insolubles de carbonato cálcico, hecho que se ve favorecido por la presencia de hidróxido de calcio (portlandita) insoluble en el medio.

La presencia de sales solubles puede provocar daños estructurales y estéticos, ya que durante el proceso de evaporación, estas sales generan tensiones alrededor del material más antiguo, más débil, provocando su deterioro.

En general los morteros de intervención son menos porosos que la fábrica original (piedra, ladrillo, etc.) con un coeficiente de resistencia mecánica y expansión térmica más alto que los materiales sobre los que se aplican. La práctica habitual ha puesto de manifiesto que la incorporación a la fábrica de materiales menos porosos puede ser peligroso.

Por otra parte el empleo de morteros de arena y cal tradicionales para su aplicación en las intervenciones, puede proporcionar resultados no satisfactorios por su difícil trabajabilidad, lenta y mala colocación en ambientes húmedos y su incompleta carbonatación bajo la superficie.

Para conocer la causa de estos problemas potenciales es importante entender la globalidad de los procesos físico- químicos que tienen lugar en los morteros tradicionales y actuales. El conjunto de reacciones que generan el endurecimiento de la mezcla se denomina fraguado del mortero y este proceso condiciona el comportamiento químico y físico de estos materiales, que es necesario conocer si se quiere de alguna manera controlar su durabilidad y compatibilidad a la hora de ser empleados en edificios de carácter histórico.

\section{FRAGUADO}

La hidratación y endurecimiento en morteros y hormigones es uno de los temas más importantes en el estudio de este tipo de materiales, su importancia se ha puesto de manifiesto en un gran número de trabajos efectuados; sobre todo desde el descubrimiento del cemento portland.

Al ponerse en contacto todos los componentes que forman parte del mortero tiene lugar una serie de reacciones de tipo físico-químicas, que es a lo que se denomina fraguado del mortero. Este proceso conlleva un desprendimiento de calor, que provoca contracciones y dilataciones durante el enfriamiento, causantes de las tensiones internas que se originan en su seno.

Aunque la mayoría de los morteros tradicionales están elaborados mayoritariamente con cal viva o yeso y a lo sumo cal hidráulica, el estudio que se propone en un primer momento se ha llevado a cabo sobre morteros modernos ( procesos mejor estudiados ) ya que su análisis puede ayudar a conocer mejor los cambios físico-químicos que tienen lugar en estos materiales y el porqué de su incompatibilidad con los morteros de yeso y cales tradicionales.

Los procesos físico-químicos del fraguado son dos fundamentalmente: la hidratación (proceso químico) y el endurecimiento (proceso físico). Estos mecanismos se van a estudiar de manera independiente aunque en realidad su evolución se da simultáneamente.

\section{Hidratación}

El cemento se forma al calcinar calizas y material arcilloso, y en el horno de calcinación se forman los siguientes compuestos:

- silicato bicálcico $\left(\mathrm{SC}_{2}\right)$. - $2 \mathrm{CaO} \mathrm{SiO}_{2}$ - belita

- silicato tricálcico $\left(\mathrm{SC}_{3}\right)$. - $3 \mathrm{CaO} \mathrm{SiO}_{2}$-alita

- aluminato tricálcico $\left(\mathrm{C}_{3} \mathrm{~A}\right)$.- $3 \mathrm{CaO} \cdot \mathrm{Al}_{2} \mathrm{O}_{3}$ -

- en menor proporción ferrito aluminato tretacálcico

( $\mathrm{C}_{4} \mathrm{AF}$ ).- $4 \mathrm{CaO} \mathrm{Al}_{2} \mathrm{O}_{3} \mathrm{Fe}_{2} \mathrm{O}_{3}$-brownmillerita

Cuando estos componentes se ponen en contacto con el agua, se forman una serie de productos de composición y morfología variable, siendo los más importantes los silicatos cálcicos hidratados ( $\mathrm{SCH}$ ), la portlandita y el trisulfatoaluminato cálcico (etringuita).

Las reacciones que tienen lugar durante la hidratación del cemento son las siguientes:

$2\left\{3 \mathrm{CaO} \mathrm{SiO}_{2}\right\}+6 \mathrm{H}_{2} \mathrm{O} \rightarrow 3 \mathrm{CaO} \cdot 2 \mathrm{SiO}_{2} \cdot 3 \mathrm{H}_{2} \mathrm{O}+3 \mathrm{Ca}(\mathrm{OH})_{2}+\mathrm{Q}$

$2\left\{2 \mathrm{CaO} . \mathrm{SiO}_{2}\right\}+4 \mathrm{H}_{2} \mathrm{O} \rightarrow 3 \mathrm{CaO} \cdot 2 \mathrm{SiO}_{2} \cdot 3 \mathrm{H}_{2} \mathrm{O}+\mathrm{Ca}(\mathrm{OH})_{2}+\mathrm{Q}$

SILICATOS CÁLCICOS + AGUA $\rightarrow$ GEL DE TOBERMORITA+ PORTLANDITA + CALOR

$3 \mathrm{CaO} . \mathrm{Al}_{2} \mathrm{O}_{3}+6 \mathrm{H}_{2} \mathrm{O} \rightarrow 3 \mathrm{CaO} . \mathrm{Al}_{2} \mathrm{O}_{3} \cdot 6 \mathrm{H}_{2} \mathrm{O}+\mathrm{Q}$

$4 \mathrm{CaO} . \mathrm{Al}_{2} \mathrm{O}_{3} \cdot \mathrm{Fe}_{2} \mathrm{O}_{3}+\mathrm{n} \mathrm{H}_{2} \mathrm{O} \rightarrow 3 \mathrm{CaO} \cdot \mathrm{Al}_{2} \mathrm{O}_{3} \cdot 6 \mathrm{H}_{2} \mathrm{O}+$ $\mathrm{CaO} . \mathrm{Fe}_{2} \mathrm{O}_{3}-\mathrm{nH}_{2} \mathrm{O}+\mathrm{Q}$

ALUMINATOS+FERRITOS+AGUA $\rightarrow$ ALUMINATOS HIDRATADOS+FERRITO HIDRATADO + CALOR

$\mathrm{CaO}+\mathrm{H}_{2} \mathrm{O} \rightarrow \mathrm{Ca}(\mathrm{OH})_{2}+\mathrm{Q}$

ÓXIDO DE CALCIO (LIBRE) +AGUA $\rightarrow$ PORTLANDITA+CALOR 
$\mathrm{MgO}+\mathrm{H}_{2} \mathrm{O} \rightarrow \mathrm{MgO}(\mathrm{OH})_{2}+\mathrm{Q}$

ÓXIDO DE MAGNESIO + AGUA $\rightarrow$ BRUCITA + CALOR

$\left.3 \mathrm{CaO} . \mathrm{Al}_{2} \mathrm{O}_{3 .+}+3 \mathrm{CaSO}_{4} 2 \mathrm{H}_{2} \mathrm{O}\right\}+25 \mathrm{H}_{2} \mathrm{O} \rightarrow$

$3 \mathrm{CaO} . \mathrm{Al}_{2} \mathrm{O}_{3} .3 \mathrm{CaSO}_{4} .31 \mathrm{H}_{2} \mathrm{O}$

ETRINGUITA

Atendiendo a las reacciones anteriores se observa que el cemento al combinarse con el agua generan portlandita $\mathrm{Ca}(\mathrm{OH})_{2}$, con desprendimiento de calor; además se forman otros compuestos: gel de tobermorita, aluminatos hidratados+ferrito hidratado, brucita, etringuita ( también llamada sal de Candlot o bacilo de Michäelis) y portlandita (procedente del $\mathrm{CaO}$ libre).

El producto de la hidratación recibe el nombre de "gel de cemento" y la fracción de pasta que no esta ocupada por dicho gel se denomina "espacio capilar". En el interior del gel se encuentran los denominados "poros del gel" de tamaño inferior a los capilares.

El proceso de hidratación tiene lugar en varias fases:

\section{Reacción inicial}

Se produce inmediatamente después de la reacción del cemento con el agua. Una vez que el cemento y el agua se ponen en contacto, y durante el periodo de amasado, se producen reacciones químicas muy rápidas. Transcurridos aproximadamente 5 minutos, la velocidad de reacción decae y la masa permanece en estado plástico. La cantidad de cemento consumida en esta fase es aproximadamente del $1 \%$ y los productos de la reacción (sólidos e insolubles) se adhieren a la superficie de los granos del cemento; estos granos permanece virtualmente igual, conservando su forma original.

\section{Periodo latente}

Se prolonga durante algunas horas, y en él no se producen reacciones químicas, permaneciendo la masa en estado plástico.

\section{Periodo de transmisión}

Formación de unos compuestos que contribuyen al endurecimiento de la pasta del mortero " fin del fraguado". El producto sólido de estas reacciones, que es el denominado "gel del cemento", tiende a llenar los espacios capilares de la pasta fresca reduciendo su volumen y tamaño.

Durante este proceso los aluminatos tricalcicos, reaccionan rápidamente con el agua originando los aluminatos tetracálcicos hidratados metaestables, que, a temperatura ambiente, terminan transformandose en estructuras más estables (Corstanje et al, 1974).

Con respecto a la hidratación del ferritoaluminato tetracálcico hay que indicar que reaccionan muy rápidamente en presencia del agua para dar hidratos análogos a los del aluminatos tricalcicos, si bien es- tos hidratos pueden aparecer como una solución sólida de aluminatos tetracálcicos hidratados y ferritoaluminatos tetracálcicos hidratados.

La hidratación de los silicatos tricalcicos puede verse retardada cuando existan aluminatos tricálcicos en el medio; el gran consumo de cal durante la hidratación de estos últimos compuestos hace que decrezca su velocidad de formación. Si además en el medio existe yeso, la formación de la etringuita hace que su hidratación comience más tarde.

Según Locher (1980) el efecto retardante del yeso se debe a su influencia sobre el tipo de producto que se va a formar. Si no existe yeso la tendencia es a formar cristales tabulares de aluminato tetracálcico hidratado; en caso contrario, se dan morfologías granuladas en la superficie de los granos de etringuita. Las primeras formas dan una estructura rígida con un fraguado rápido de la pasta y en las segundas el fraguado comienza después del periodo latente, como consecuencia de la recristalización de la etringuita a partir de cristales más pequeños anteriormente formados.

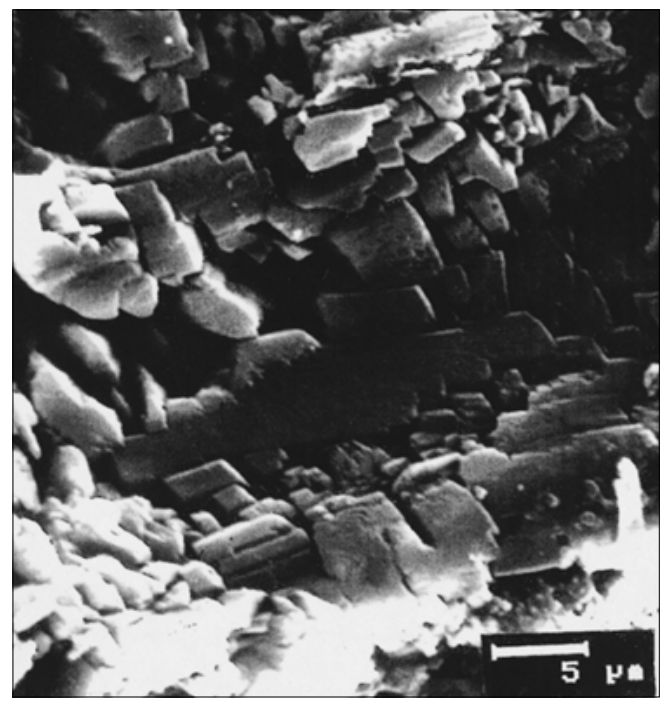

Yeso de alteración desarrollada en la superficie de un mortero de cal.

Cuando el ión sulfato desaparece, la etringuita se transforma en monosulfatoaluminato de calcio hidratado, más permeable, lo que hace que comience la hidratación de los silicatos tricálcicos. En el avance de la hidratación de los aluminatos y silicatos cálcicos, los productos generados rellenan los huecos dejados por esta estructura, incrementando la resistencia.

Según lo anteriormente indicado, si en el medio existe gran cantidad de aluminatoe tricálcicos es muy probable que aparezca la etringuita.

La hidratación de los silicatos tricálcicos, es más rápida que la de los silicatos bicálcicos pero los productos resultantes son los mismos y constituyen la fase más amorfa de los silicatos cálcicos hidratados, su formación se produce en 5 fases (Kondo y Diamond, 1974), atendiendo a su velocidad de formación, que 
es variable según los distintos estadios. Constituyen aproximadamente el $75 \%$ de la masa total del cemento. Los silicatos cálcicos hidratados, por su analogía con el mineral natural tobermorita, se han denominado "gel de tobermorita".

Los geles de tobermorita procedentes de las reacciones de los silicatos tricálcicos y bicálcicos son idénticos en difracción y microscopía electrónica; sin embargo dicho gel no está integrado solamente por estos dos compuestos, sino que comprende una serie de hidratos de composiciones variables que constituyen un subgrupo de compuestos dentro de ella. La composición del gel cambia en el curso de la hidratación; Kantro et al (1962) observaron relaciones molares $\mathrm{CaO} / \mathrm{SiO}_{2}$ que varían de 1.39 a 1.73 .

Por otra parte en los geles de tobermorita, debido a su carácter hidrófilo, también varia la relación $\mathrm{H}_{2} \mathrm{O} / \mathrm{SiO}_{2}$; dichos compuestos tras varios procesos de secado pierden agua hasta un mínimo que es imposible eliminar.

Powers y Brownyard (1952) han demostrado que la superficie específica y la porosidad de la pasta de tobermorita desempeñan papeles decisivos en las propiedades mecánicas, cambios de dimensión y permeabilidad del agua.

Según Kondo y Daimond (1974), al ponerse estos compuestos en contacto con el agua, en la superficie se forma una pequeña capa de hemihidrato donde la fase acuosa se enriquece en cal hidrolizada; un segundo hidrato germina sobre esta primera capa menos impermeable que no es más que hidróxido de calcio en forma metaestable.

Para Ludwig (1972), los silicatos tricálcicos se disuelven inmediatamente y los silicatos cálcicos hidratados se generan inmediatamente en la periferia de los granos, la lixiviación del calcio se hace a través de esa membrana por cambio de protones con el agua y su velocidad de hidratación se ralentiza debido a la existencia de esta capa. Los silicatos cálcicos hidratados lixiviados de su cal permaneciendo " in situ" se denominan hidrato interno, mientras el silicato cálcico hidratado precipitado en el exterior de los granos, a la vez que la portlandita, constituye el hidrato externo.

La portlandita procede de la hidratación de los silicatos cálcicos, está relacionada con el final del periodo latente, apareciendo al principio como pequeños cristales en la superficie de todos los productos. Estos núcleos crecen con el tiempo dando cristales grandes bien facetados. La concentración de calcio en la disolución es la responsable de su morfología.

Si en el medio existe una sobresaturación de sílice este proceso se retarda, por esta razón en el cemento portland el proceso de formación de la portlandita es menor con respecto a soluciones más ricas en componentes carbonatados.
Existen varias teorías acerca de la hidratación: una teoría considera que el proceso tiene lugar por un mecanismo llamado "vía solución" con disolución previa y posterior precipitación, modelo de Chatelier (Kittl, et al, 1980) y la otra propone que el mecanismo de crecimiento, según Novo Fernández, (1988) de los silicatos cálcicos hidratados es de tipo topoquímico donde las reacciones se producen en estado semisólido. Lo más probable es ambos proceso se produzcan simultáneamente, sin embargo esta controversia en la actualidad no está totalmente resuelta.

La mayor parte de los productos de hidratación del cemento están imperfectamente cristalizados y, por ello, sus temperaturas y presiones de disociación no son constantes. Por otra parte, una porción de agua liquida queda adherida a esos compuestos mediante fuerzas de absorción y capilares, agua que no puede eliminarse solo por evaporación.

\section{Endurecimiento}

El endurecimiento de la pasta se considera como una consolidación de un esqueleto mineral formado por granos anhidros y portlandita, etringuita e hidroaluminatos cálcicos formados en los primeros instantes de la hidratación. La consolidación se efectuará por el desplazamiento progresivo de los espacios disponibles del gel de silicato cálcico hidratado y la portlandita, ésta aparece en el comienzo de la hidratación como plaquetas hexagonales condicionando el espacio existente.

Por tanto en la fase inicial el elemento implicado directamente es el aluminato tetracálcico, mientras los silicatos tetracálcicos reaccionan en una pequeña proporción para dar portlandita. Según Locher (1980), la resistencia mecánica comienza al concluir la reacción del aluminato tetracálcico con el sulfato cálcico, es decir cuando ya se han formado la etringuita primaria, aluminatos hidratados y /o monosulfatos, los cuales dan una pequeña resistencia a la pasta.

De acuerdo con las observaciones llevadas a cabo en el microscopio electrónico, la fase de silicatos cálcicos hidratados comienza a desarrollarse como microcristales alargados alrededor de los granos, posteriormente adquieren un hábito acicular (de longitud I micra). Estas agujas se fusionan cuando entran en contacto formando un amasijo de fibras reticulares, esto genera una red de espacios vacíos que son rellenados por otros productos de hidratación durante el envejecimiento de la pasta.

Los aluminatos tricálcicos se transforman por efecto de la hidratación en cristales cúbicos de $\mathrm{C}_{3} \mathrm{AH}_{6}$ y la etringuita aparece en forma de cristales prismáticos hexagonales en los espacios disponibles.

La hipótesis más acertada para el caso de los $\mathrm{SCH}$ es la topoquímica, en la que aparece una primera mem- 
brana de gel amorfo, coloidal y de estequiometría variable. Con el avance de la hidratación el gel puede ir adoptando morfologías variables:

I. estructura fibroso/acicular radiante desde las partículas del cemento,

2. forma reticular, alveolar, con menos desarrollo aéreo de las morfologías,

3. de morfología no definida de forma irregular rellenando huecos,

4. morfología amorfa, de grano muy fino; se forman en la interfase parte hidratada-grano de cemento, ocupando el espacio producido por la disolución del grano.

Las tres morfologías primeras son productos externos al grano del cemento, formados en los poros y la última corresponde a las partes más internas del grano.

La etringuita y los aluminatos-ferritos tetracálcicos se forman más probablemente por un mecanismo "vía solución" ; este mecanismo de disolución-precipitación justifica las morfologías tan variables con que se presentan los aluminatosferritos tetracálcicos.

\section{Carbonatación}

Es el proceso fisicoquímico del fraguado más importante en morteros antiguos y el único que se produce en morteros no hidráulicos, por tanto los procesos reactivos que lo generan serán los factores que determinan sus características físico-químicas y tecnológicas.

La carbonatación tiende a modificar la estructura de la pasta tanto, desde el punto de vista físico como químico, esto se traduce en la modificación de sus cualidades: resistencia mecánica, porosidad, permeabilidad, durabilidad frente a los agentes salinos, etc.

La acción del anhidrido carbónico sobre los compuestos que constituyen el mortero tiene una serie de efectos que se pueden sintetizar en dos puntos fundamentalmente: acción neutralizadora y formación de compuestos más cristalinos.

El compuesto que primero reacciona con el $\mathrm{CO}_{2}$ es la portlandita, $\mathrm{Ca}(\mathrm{OH})_{2}$, constituyente fundamental de los morteros de cal, y le siguen los aluminatos y ferritoaluminatos cálcicos (Leber, Blakey; 1956 y Veerberk; 1958), para el caso de morteros hidráulicos y de cemento portland.

El proceso de carbonatación se produce de la forma indicada a continuación:

$\mathrm{Ca}(\mathrm{OH})_{2}+\mathrm{CO}_{2} \leftrightarrow \mathrm{CO}_{3} \mathrm{Ca}+\mathrm{H}_{2} \mathrm{O}$

Esta reacción es la primera en producirse y a partir de la portlandita se forma el carbonato cálcico, en forma de calcita, aragonito o vaterita con desprendimiento de agua.

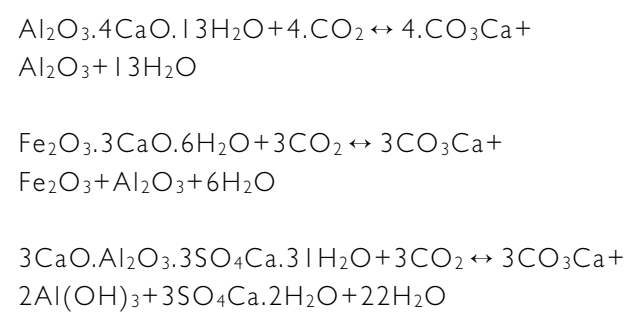

Estas tres últimas reacciones se forman a partir de compuestos poco cristalinos generando compuestos de $\mathrm{CO}_{3} \mathrm{Ca}, \mathrm{Al}_{2} \mathrm{O}_{3}$ y $\mathrm{Fe}_{2} \mathrm{O}_{3}$ más cristalinos.

$2 \mathrm{SiO}_{2} .3 \mathrm{CaO} .3 \mathrm{H}_{2} \mathrm{O}+3 \mathrm{CO}_{2} \leftrightarrow 3 \mathrm{CO}_{3} \mathrm{Ca}+2 \mathrm{SiO}_{2}+$ $3 \mathrm{H}_{2} \mathrm{O}$

Esta última reacción se produce a partir del gel de tobermorita (silicato hidratado) que genera sílice y carbonato cálcico.

En la mayor parte de las reacciones indicadas anteriormente se origina y/o interviene el carbonato cálcico, es por tanto el comportamiento de este compuesto el que marcará de forma importante las características de la pasta endurecida.

El efecto del $\mathrm{CO}_{2}$ en los distintos compuestos del cemento portland se recoge a continuación.

I. Acción del $\mathrm{CO}_{2}$ sobre la portlandita. Como se ha indicado anteriormente la portlandita aparece como placas hexagonales y es el primer compuesto que se forma en la pasta hidratada.

La calcita se forma en presencia de agua tras la disolución del $\mathrm{CO}_{2}$ y la posterior precipitación del carbonato cálcico tiene lugar en zonas próximas a la ubicación inicial de la portlandita, rellenando oquedades. Este proceso trae consigo una reducción de volumen.

Al principio de la carbonatación y dado el carácter básico de la pasta se forma la vaterita (formas menos cristalinas) en forma de esferulos junto al aragonito si en el medio existe sulfatos y sales de bario, estroncio y magnesio. Con el tiempo, y en presencia de disoluciones de carbonato cálcico, la vaterita y el aragonito se van transformando en calcita (forma más cristalina).

2. Acción del $\mathrm{CO}_{2}$ sobre los aluminatos tricálcicos. A diferencia de los silicatos cálcicos estos compuestos se disuelven totalmente en agua, desapareciendo durante el proceso de hidratación. La presencia de portlandita disuelta en el agua y la existencia de yeso, hace que el proceso de formación de estos compuestos se complique.

La dinámica de formación tiene lugar en tres fases.

\section{$I^{a}$ Fase}

$\mathrm{C}_{3} \mathrm{~A}+3 \mathrm{SO}_{4}=+\mathrm{Ca}^{++}+32 \mathrm{H}_{2} \mathrm{O} \rightleftarrows \mathrm{C}_{3} \mathrm{~A} \cdot \mathrm{SO}_{4} \mathrm{Ca} \cdot 32 \mathrm{H}_{2} \mathrm{O}$ 
En la primera fase se forma la etringuita, que se encuentra normalmente rellenando los huecos formados después del proceso de endurecimiento. Su formación se debe al ataque que sufren los aluminatos tricálcicos de la pasta por parte de los sulfatos. Esta sal puede transformarse en thaumasita $\left(\mathrm{CaCO}_{3}\right.$. Ca$\mathrm{SO}_{4} \cdot \mathrm{CaSiO}_{3}$. I $5 \mathrm{H}_{2} \mathrm{O}$ ) y es frecuente encontrarla en morteros con impurezas de material arcilloso y normalmente aparece alrededor de la superficie de los granos. Esta sal es muy expansiva, algunos autores cifran en $227 \%$ el incremento sobre el volumen inicial del $\mathrm{AC}_{3}$.

Los aluminatos restantes se hidratan en presencia de la portlandita.

\section{$2^{a}$ Fase}

$\mathrm{C}_{3} \mathrm{~A}+\mathrm{Ca}(\mathrm{OH})_{2}+12 \mathrm{H}_{2} \mathrm{O} \rightleftarrows \mathrm{C}_{4} \mathrm{AH}_{13}$

Esta segunda reacción genera aluminato hexagonal y supone una reducción de volumen.

\section{$3^{a}$ Fase}

Finalmente, el aluminato cálcico hidratado reacciona con el trisulfoaluminato cálcico para dar un monosulfatoaluminato cálcico según la siguiente reacción:

$\mathrm{C}_{3} \mathrm{~A} .3 \mathrm{SO}_{4} \mathrm{Ca} .32 \mathrm{H}_{2} \mathrm{O}+2 \mathrm{C}_{4} \mathrm{AH} \mathrm{H}_{3} \rightleftarrows 3 \mathrm{C}_{3} \mathrm{~A} . \mathrm{SO}_{4} \mathrm{Ca}$. $12 \mathrm{H}_{2} \mathrm{O}+\mathrm{Ca}(\mathrm{OH})_{2}+2 \mathrm{OH}_{2} \mathrm{O}$

De esto se deduce que los aluminatos cálcicos dan lugar a tres compuestos que pueden estar presentes en la pasta del cemento: aluminato cálcico hexagonal, trisulfoaluminato cálcico (etringuita) y monosulfoaluminato cálcico.

Los aluminato se encuentran en baja proporción en los morteros hidráulicos antiguos, pero su hidratación es muy rápida pudiendo afectar a la mezcla final de mortero.

3. Acción del $\mathrm{CO}_{2}$ sobre el $\mathrm{C}_{4} \mathrm{AH}_{13}$. Estas sustancias al reaccionar con el dióxido de carbono generan unos compuestos denominados carboaluminatos, que se forman según la siguiente reacción:

$\mathrm{C}_{4} \mathrm{AH}_{13}+\mathrm{CO}_{2} \rightleftarrows \mathrm{C}_{3} \mathrm{~A} . \mathrm{CO}_{3} \mathrm{Ca} .11 \mathrm{H}_{2} \mathrm{O}+2 \mathrm{H}_{2} \mathrm{O}$

Esta reacción es muy rápida al ponerse en contacto con el $\mathrm{CO}_{2}$; sin embargo la presencia de este compuesto solo se da en pastas débilmente carbonatadas por acción natural. En el caso de que la acción del $\mathrm{CO}_{2}$ prosiga, se descompone en calcita y gibsita según la siguiente reacción:

$\mathrm{C}_{3} \mathrm{ACO}_{3} \mathrm{Ca} .11 \mathrm{H}_{2} \mathrm{O}+3 \mathrm{CO}_{2} \rightleftarrows 4 \mathrm{CO}_{3} \mathrm{Ca} .+$ $2 \mathrm{Al}(\mathrm{OH})_{3}+8 \mathrm{H}_{2} \mathrm{O}$

4. Acción del $\mathrm{CO}_{2}$ sobre la etringuita (sulfoaluminatos cálcicos). La acción del dióxido sobre este compues- to actúa descomponiendolo de acuerdo con la siguiente ecuación:

$\mathrm{C}_{3} \mathrm{~A} .3 \mathrm{SO}_{4} \mathrm{Ca} .32 \mathrm{H}_{2} \mathrm{O}+3 \mathrm{CO}_{2} \rightleftarrows 3 \mathrm{CO}_{3} \mathrm{Ca}+2 \mathrm{Al}(\mathrm{OH})_{3}$ $+\mathrm{SO}_{4} \mathrm{Ca}_{2} \mathrm{H}_{2} \mathrm{O}+23 \mathrm{H}_{2} \mathrm{O}$

En este caso el carbonato cálcico aparece como aragonito y esta reacción lleva consigo una reducción de volumen y producción de humedad.

5. Acción del $\mathrm{CO}_{2}$ sobre el monosulfato. Los compuestos se desdoblan de forma parecida a la etringuita:

$\mathrm{C}_{3} \mathrm{~A} . \mathrm{SO}_{4} \mathrm{Ca} .12 \mathrm{H}_{2} \mathrm{O}+3 \mathrm{CO}_{2} \rightleftarrows 3 \mathrm{CO}_{3} \mathrm{Ca}+2 \mathrm{Al}(\mathrm{OH})_{3}+$ $\mathrm{SO}_{4} \mathrm{Ca} .2 \mathrm{H}_{2} \mathrm{O}+7 \mathrm{H}_{2} \mathrm{O}$

6. Acción del $\mathrm{CO}_{2}$ sobre los silicatos cálcicos hidratados. Teniendo en cuenta la estructura de la tobermorita estable, parece evidente que tendrá que verificarse la siguiente reacción:

$\mathrm{Ca}_{2}\left[\mathrm{SiO}_{2}(\mathrm{OH})_{2}\right]_{2} \cdot \mathrm{CaO} \cdot \mathrm{H}_{2} \mathrm{O}+\mathrm{CO}_{2} \rightleftarrows \mathrm{CO}_{3} \mathrm{Ca}+2 \mathrm{Ca}$

$\left[\mathrm{SiO}_{2}(\mathrm{OH})_{2}\right]+\mathrm{H}_{2} \mathrm{O}$

Según Bernal (1952), si el $\mathrm{CaO} . \mathrm{H}_{2} \mathrm{O}$ de su estructura está débilmente unido, hasta el punto de poder ser eliminada por desecación, el $\mathrm{CO}_{2}$ actuará sobre este compuesto. Si por otra parte el ácido carbónico tiene afinidad más por la cal que el ácido silícico, parece lógico que se produzca:

$\mathrm{Ca}\left[\mathrm{SiO}_{2}(\mathrm{OH})_{2}\right]+\mathrm{CO}_{2}+\mathrm{H}_{2} \mathrm{O} \rightleftarrows \mathrm{CO}_{3} \mathrm{Ca}+\mathrm{SiO}_{2}(\mathrm{OH})_{2}$

Teniendo en cuenta la reacción anterior; se origina carbonato cálcico y sílice amorfa. El hecho de interpretar que la sílice queda como $\mathrm{SiO}_{2}(\mathrm{OH})_{2}$ en lugar de $\mathrm{SiO}_{2}$, se debe a que Meyers (1949) manifiesta que la silice separada es amorfa y capaz de intervenir en las reacciones "árido-álcalis".

En condiciones atmosféricas la velocidad de carbonatación es rápida, pero ésta va disminuyendo a medida que comienzan a formarse los cristales de calcita a partir de la portlandita. La porosidad y permeabilidad desempeñan un papel importante en el transporte del $\mathrm{CO}_{2}$, por tanto si éstas se reducen, se hace cada vez más difícil la entrada del $\mathrm{CO}_{2}$ al interior y la salida del agua hacia el exterior.

El agua de infiltración, aunque favorece la disolución del carbonato cálcico, puede facilitatar el transporte de este gas y provocar la formación de un compuesto no carbonatado, como $\mathrm{Ca}(\mathrm{OH})_{2} \cdot 2 \mathrm{H}_{2} \mathrm{O}$, cuya proporción en algunos casos puede llegar hasta el $30 \%$ del total de la cal.

La velocidad de carbonatación también depende de la naturaleza del aglomerante, composición química, finura, relación agua/cemento, compacidad, estado de hidratación de la pasta, naturaleza del árido y condiciones atmosféricas (Temperatura ambiente y humedad relativa). 
Investigaciones llevadas a cabo en este sentido han demostrado que las humedades óptimas para la carbonatación están comprendidas entre 45-75\%, para humedades por encima de este valor, los poros del material se colmatan impidiendo la difusión del $\mathrm{CO}_{2}$ hacia el interior; por contra, si la humedad está por debajo del $25 \%$ la carbonatación se reduce como consecuencia de la falta de agua líquida necesaria para que se produzca la reacción.

La velocidad de carbonatación como se ha indicado antes, depende de la composición del árido; así los morteros con material puzolánico son los que más rápidamente se carbonatan. Esto está relacionado con su porosidad siendo los áridos más ligeros los que más favorecen el proceso de carbonatación.

La granulometría del cemento al principio no influye en la carbonatación pero, pasado un año se hace más sensible y la carbonatación es más rápida cuanto menor es la finura; aunque esta variación es apenas perceptible. En contraposición, si aumenta el contenido de cemento se produce una reducción de la porosidad y por tanto un freno en el proceso de carbonatación.

Verbeck (1958) establece que la velocidad de carbonatación debe ser inversamente proporcional a la profundidad de carbonatación

Según todo lo visto podemos concluir que la presencia del $\mathrm{CO}_{2}$ es fundamental para el proceso de carbonatación, su control condiciona la hidratación de los componentes dando como resultado comportamientos mecánicos diferentes en la pasta del mortero.

El deterioro del ligante, comienza cuando cesa el proceso de carbonatación y, en presencia de agua ionizada (agua de lluvia), se transforma en bicarbonato, que sí es soluble. Este proceso reactivo puede ser controlado mientras en el medio exista portlandita libre, que actúa como neutralizador. Sin embargo este proceso es lento y no afecta de manera importante a la integridad del mortero.

En una situación ideal, la carbonatación debe producirse de forma lenta para evitar la excesiva hidratación de los silicatos. En morteros antiguos se dan estas condiciones como consecuencia de la formación de una capa superficial impermeable que facilita la estabilidad de estas fases (silicatos cálcicos hidratados).

\section{REACCIÓN ÁRIDO-ÁLCALIS}

Hasta mediados de los años 50 era admitida la hipótesis de Feret, según la cual la naturaleza mineralógica de los áridos no tenia ninguna influencia en el comportamiento de los morteros y hormigones endurecidos.

Stanton (1940) fue el primer autor que atribuye los defectos de una presa a la interacción entre los áridos de naturaleza silícea y los álcalis del cemento. Posteriormente Aardt y Visser (1997), Cole et al (1981), Wary y Cole (1982) y Soriano (1981, 1987, 1989), entre otros, han estudiado las reacciones de interacción entre ciertos tipos de áridos y los componentes de la fase intersticial del hormigón, estableciendo que se generan nuevas fases silicatadas que pueden influir en la durabilidad de estos materiales.

La reacción de ciertos áridos silíceos con los álcalis del cemento originan unos geles de sílice que, generalmente tienen una acción osmótica en presencia de agua, y que, por absorción de ésta, aumentan de volumen y generan importantes presiones disruptivas. Existen tres factores fundamentales para que la reacción se desarrolle y son: la existencia de estos áridos reactivos (con sílice amorfa), la presencia de álcalis y agua abundante en el medio.

Los áridos susceptibles de producir estas reacciones son los de tamaño intermedio (2 a 8 mm). Los áridos de tamaño menor a $2 \mathrm{~mm}$ al presentar una elevada superficie reactiva, pueden llegar a desaparecer del medio. Favorecen esta reacción los minerales opalinos (andesitas, riolitas, pizarras, filitas y ceolitas de tipo heulandita). El esquema de este proceso viene dado en la siguiente reacción:

$$
\begin{gathered}
\mathrm{SiO}_{2}+\mathrm{Na}(\mathrm{OH})+\mathrm{Ca}(\mathrm{OH})_{2}+\mathrm{H}_{2} \mathrm{O} \rightarrow \\
\mathrm{n}_{1} \mathrm{Na}_{2} \mathrm{O} . \mathrm{nCaO} \cdot \mathrm{nSiO}_{2} \mathrm{n}_{4} \mathrm{H}_{2} \mathrm{O}
\end{gathered}
$$

El gel resultante será expansivo en función del contenido en $\mathrm{CaO}$.

Hadley (1961) observó una reacción de carácter disruptivo similar a la de Stanton, entre áridos de naturaleza dolomítica y los álcalis del cemento.

En este caso, al ponerse estos áridos en contacto con soluciones alcalinas, generan brucita (hidróxido de magnesio) e hidróxidos alcalinos según los siguientes procesos:

$\left(\mathrm{CO}_{3}\right)_{2} \mathrm{CaMg}+2 \mathrm{NaOH} \rightarrow \mathrm{Mg}(\mathrm{OH})_{2}+\mathrm{CO}_{3} \mathrm{Ca}+$ $\mathrm{CO}_{3} \mathrm{Na}_{2}$

\section{$\mathrm{CO}_{3} \mathrm{Na}_{2}+\mathrm{Ca}(\mathrm{OH})_{2} \rightarrow \mathrm{CO}_{3} \mathrm{Ca}+2 \mathrm{NaOH}$}

La brucita puede aparecer bien en las zonas de contacto entre el árido y la pasta, o bien en el mismo árido formando un silicato de magnesio. Este proceso de dolomitización genera un incremento de porosidad y debilitamiento de la unión pasta-árido, pero en ningún caso presenta carácter expansivo.

Aardt y Visser (1977) comprobaron que la reacción entre el hidróxido de calcio y determinados tipos de áridos, con arcilla y feldespatos, da lugar a la formación de hidrogranates, silicato cálcico y álcalis libres; comprobaron que el ataque de feldespatos y arcillas puede considerarse como un tipo de reacción cemento-árido que, teniendo un carácter más amplio que la reacción árido-álcalis, también puede llevar a la destrucción del hormigón. 
Cole et al (1981) observaron el desarrollo de ceolitas alrededor de los áridos por medio del microscopio electrónico (SEM), formando unos anillos oscuros y en su interior unos depósitos blanquecinos; los poros aparecían rodeados de unas sustancias transparentes semejantes a las observadas en los áridos.

Soriano (1997), Alonso et al, (1998) y Soriano y García Calleja (1989, 1993) entre otros han puesto de manifiesto mediante microscopía electrónica de barrido, la existencia de reacciones de interacción entre determinados tipos de áridos y el hidróxido de calcio liberado durante la hidratación del cemento portland. Como consecuencia del intercambio catiónico que tiene lugar entre los áridos y la solución, se produce una pérdida de sódio, magnesio, azufre y potásio y una ganancia de calcio y en ocasiones de hierro. Este intercambio se ve favorecido por la elevada basicidad del hormigón y la necesidad de los minerales a adaptarse a las nuevas condiciones del medio.

Biczok (1978) afirma que cuando un grano de árido (de composición silícea en estado inestable) se pone en contacto con una disolución de álcalis semejante a la que puede existir en un mortero, la capa más externa del grano comienza a ser atacada y esto se manifiesta por un reblandecimiento y aumento del volumen del mismo. El proceso se desarrolla como si los grupos de sílice, que forman el grano, se "abrieran" para incorporar las moléculas de agua junto con los compuestos de la disolución alcalina. Esto trae consigo un aumento de volumen que puede generar presiones de hasta $10 \mathrm{kp} / \mathrm{cm}^{2}$ suficientes para fisurar el material.

Veronelli (1978) considera que el proceso tiene un carácter osmótico y, ocurre cuando el hidróxido de calcio, liberado en la hidratación del cemento, reacciona con la silice atacada previamente por los álcalis, formandose una capa fina de silicato cálcico insoluble sobre la masa del silicato alcalino. Esta capa actúa como una membrana semipermeable que deja pasar sólo el agua, con lo que aumenta la presión en el interior del grano.

La reacción árido-álcalis justifica los daños a través del proceso osmótico.

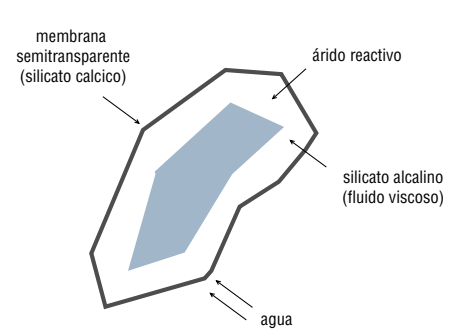

La diferencia entre una hipótesis u otra radica en que el primer caso, las presiones se producirían en un volumen realmente amplio de la masa del mortero, ya que es el fluido viscoso el que transmite las presiones y en el segundo caso, el aumento de presión se produciría en el árido y sólo en sus inmediaciones se detectarían los efectos de ese aumento de volumen.

Los iones de potásio y sódio más la cal liberada por el cemento y áridos reactivos (feldespatos, micas, etc) forman un gel (cal-álcalis y sílice); que se manifiesta como grietas en el contorno de los áridos. Cuando estas grietas aparecen es difícil identificar si su origen se debe al efecto de estos compuestos o es una fisuración propia del árido originada durante el proceso de machaqueo.

Los álcalis que forman este gel pueden provenir:

- del agua de amasado,

- de los procesos de reacción durante el fraguado del mortero,

- del ataque de la solución alcalina a los áridos reactivos originando un gel álcalis-silice en la superficie de los áridos (gel no expansivo).

Este gel actúa como una membrana de separación entre la solución alcalina y ácido silícico, que puede reaccionar y absolver iones calcio y sodio. Se pueden dar dos situaciones:

a) Medio muy rica en cal; en este caso se forma un gel de cal-álcalis-silice.

b) Medio dominado por los álcalis, se origina un gel alcalino de silice que sí es expansivo.

Este gel absorbe agua de la pasta y se introduce por el interior de las fisuras generando presiones.

Investigaciones llevadas a cabo por Fontao et al (1994) empleando el acetato de uranilo permite identificar las alteraciones en áridos. Dicho gel puede originar un intercambio iónico con $\mathrm{UO}_{2}{ }^{+2}$, originando un silicato con iones uranilo. La composición de este gel va a depender del tanto por ciento de sílice reactiva así como de los álcalis disponibles.

Este método también permite desechar ataque por sulfatos (problemas de etringuita) ya que si los efectos son los mismos mediante este método estos últimos dan resultados negativos.

Los álcalis que intervienen en este fenómeno proceden en su mayor parte del cemento $(\sim 2 \%)$, la solución puede estar en evitar el empleo del cemento con alto contenido en estos elementos.

La reacción entre la cal y los áridos de naturaleza silícea (en morteros de cal) es un proceso lento, que se ha observado en morteros antiguos (Ontiveros, 1997); este fenómeno se pone de manifiesto por la existencia de ciertas fases en la zona de contacto entre el ligante (la cal) y el árido que indican sin ninguna duda la reacción entre ambos. Estos compuestos, tras su hidratación contribuyen al endurecimiento del mortero y facilitan la adherencia ligante-agregado, si bien teniendo en cuenta las proporciones en las que aparecen, su incidencia no debe ser importante. 
AARDT, J H P; VISSER, S. Calcium hidroxide attack on feldespars and clays: possible relevance to cement-aggregate reactions. Cem. Concr. Res., 7, 1977. Pp 643-648.

ALONSO, J L; SORIANO, J; BARBA, C; RIESGO, L. Fisuración de una presa española por reacción entre un árido granítico y el hidróxido de calcio liberado en la hidratación del cemento portland. Jornadas en español y portugués sobre estructuras y materiales (colloquia 88),2. Pp I03-127.

BERNAL. Tercel Symposium Int. de Química del Cemento, Londres, 1952, pág. 216.

BICZOK. Corrosión y protección del hormigón. Ed. Urno. Bilbao, 1978.

BRUNAUER Y WEISE. Advances in Chemistry Series, 1962.

COLE, W.F; LANCKUKI, C]. Products formed in and aged concrete. Cem. Concr. Res. 1981, II. pp 443-454.

CORSTANJE, W A; STEIN, H N; ATEVELS, J M. Hydration reactions in paste $\mathrm{C}_{3} \mathrm{~S}+\mathrm{C}_{3} \mathrm{~A}+\mathrm{CaSO}_{4}$. 2aq + water at $25^{\circ} \mathrm{C}$. Cem. Concr. Res.4 1974. Pp. $125-128$.

FERNÁNDEZ PARIS, J M. La carbonatación de la pasta hidratada del cemento portland. Interpretación físico-química. Monografía del Instituto Eduardo Torroja de la construcción y el cemento. Madrid 1973.

FONTAO I; LEIRO. A; MATEO, B; PRENDES, N (1994). Estudio de patologías del hormigón mediamte técnicas con acetato de uranilo. Boletín de la Sociedad Española de Mineralogía, 17 (1994)pp. 169-177

HADLEY, D W. Alkali reactivity of dolomite carbonate rocks. Higbway Research Board, 40; 1961. Pp462-469.

HAMADA, M. Proceeding of the fifth International Syposium of the Cheistry of cement. Tokio, 1968. Volumen III.

KANTRO, BRUNAUER Y WEISE. Advances in Chemistry Series, 1962.

KISHITANI, K. Zement Kalk Giup. 1964.

KITTL, P et AL. 7th. ICCC. Paris, VII,II 1980. Pp I4I.

KONDO, R; DAIMOND, M. Phase composition of hardened ce-

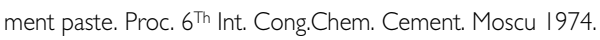

LEBER y BLAKEY. Acción del dióxido de carbono en morteros y hormigones. Journal American Concrete Institute. Septiembre, 1956. Pp. 295-308.

LOCHER F W. 7Th ICCC Paris. General Rport, V.IV, 1980.
LUDWING, U. "On the setting and hardening of cement. TonindZtg 96.1972. Pp.85-92.

MEYER, S L. Effects of carbon dioxide in hidrated cement and concrete. Rock products. Enero, 1949. Pp. 96-98.

ONTIVEROS, E; SEBASTIÁN PARDO, E. y VALVERDE, I. Deterioration in IX-XIV century arab rampart (Granada, Spain). Materals and structurs, Vol 32 Junnuary-February 1999. Pp 45-51. Rilem 159-5997/99.

NOVO FERNANDÉZ, R; DÍEZ-CASCÓN, J; GOMEZ LAÁ, G. Aspectos morfológicos de conglomerados con cenizas volantes. Congreso geológico de España, 1988, comunicaciones volumen II. Pp.286-287.

POWERS y BROWNYARD. Jour. Am. Concr. Research, 1952.

POWERS, T Y BROWNYARD, H. Studies of the physical properties of hardened portland cement paste. Journal American concrete Institute. Diciembre 1946. Pp. 469.

SORIANO CARRILLO, J. Influencia de la naturaleza mineralógica de las adiciones en el comportamiento de la pasta endurecida del cemento portland. Revista de Obras Públicas, 3195, 1981. Pp. 577-584.

SORIANO CARRILLO, J.; GARCÍA CALLEJA, MA. Áridos reactivos. Acción del hidróxido de calcio sobre áridos silicatados. III Congreso de Geoquímica de España I, 1989. Pp. 9-15.

SORIANO CARRILLO, J. El proceso de endurecimiento del cemento portland. II Congreso de geoquímica de España, 1997.pp. 279-292.

SORIANO CARRILLO, J. Reactions d'nteraction entre certains granulats et la phase interstitielle du beton. In pore Structure and materials propertiers. Chapman and hall. London, 1987. Pp. 378.

SORIANO CARRILLO, J.; GARCÍA CALLEJA, MA. Estudio geoquímico de un hormigón antiguo. $\vee$ Cong. Geoquímica España, 1993. Pp. 225-230.

VERBECK G.L. Carbonation of hidrated portland cement. Amarican Society Testing material-Special Technical Publication, 1958, pp. 214-217.

VORONELLI. Durabilidad de los hormigones. Reacción árido-alcalis. Monografias n³52 del IETcc Madrid, 1978.

WAY, SJ; COLE, WF. Calcium hidroxide attack rocks. Cem. Concr. Res. 12, 1982. Pp 611-617. 